passing round the needles are fastened to the wire banjo and provide the extension.

Those without experience of the method might well imagine this transfixion to be a septic and painful procedure. That it is neither is as astonishing as it is true.

Fracture of

the Neck of shoulder of a meat porter. It is difficult to, retain reduction. Under

the Scapula. full anæsthesia, the patient wearing a vest with arms has a plaster case applied to the chest, the plaster being well moulded over the scapula and its axillary border with the arm near the side. When the plaster has dried the arm is abducted and traction exerted on it. The plaster case is now extended to take in the arm as far as the metacarpal heads, the elbow being flexed and the wrist dorsiflexed. The case is bivalved and the upper half removed after three weeks, when massage is commenced. The rest of the case is removed two weeks later.

(a) Co-incident Nerve Injuries.-Paralysed muscles are prevented from

Complications
of Fractures

of the

Upper Limb. stretching by suitable splinting and their nutrition maintained by physiotherapy. Should signs of returning function not appear, the nerve must be explored and freed or resutured.

(b) Sepsis.-This is usually due to the fracture being an open one from the outset. Treatment consists in packing the wound with gauze and cleansing the surrounding skin. Next the edges of the wound all hopelessly damaged tissues are excised.

The wound is wiped out with ether and Carrel-Dakin tubes sewn to gauze placed in its depth. Freshly prepared Dakin solution is run in at regular intervals, until it is shown both clinically and by swab smears of the wound juices that infection has been overcome. It is sometimes amazing how little sepsis results from the gross traumatic insults which tissues sustain. The writer once removed from the knee-joint two ounces of road grit, grease and mud. The joint healed by first intention.

(c) Delayed Union.-If there be apposition and asepsis, this is best treated by a buffed leather gaiter splint lacing around the limb. Exercises are performed promoting axial pressure on the broken ends. The patient leads an open-air life with a liberal diet.

(d) Non-Union.-This will already have had treatment under the heading of delayed union. Should this fail an inlay bone graft is advised forthwith. It is usually obtained from the patient's tibia. It is to be preferred to a dowelled intramedullary peg.

\title{
THE TECHNIQUE OF THE INJECTION TREATMENT OF HYDROCELE.
}

\author{
BY RODNEY H. MAINGOT, F.R.C.S.ENG.
}

Surgeon, Royal Waterloo Hospital, London.

THE rationale of the treatment is the introduction of a sclerosing solution into the sac, sufficiently irritating to produce an aseptic, intense, inflammatory reaction of the serous lining, which reaction causes the opposing walls to adhere firmly, and since endothelium is mesodermal in origin the process of repair following this reaction 
leads to the formation of fibroblasts, and ultimately to the obliteration of the sac by fibrous tissue.

It is best to have the patient lying prone on a couch or operating table, as he is less likely to faint in this position, and the procedure of injection is facilitated for the surgeon. The requisites for the injection are : (I) A torch with a powerful light; (2) a hypodermic syringe and needle ; (3) a ro-c.c. Record syringe and a stiletted spinal puncture needle of No. 20 wire gauge with its point bevelled at an angle of $45^{\circ}$; $\stackrel{\mathbb{\Omega}}{\AA}$ (4) solution: quinine-urethane, or lithium salicylate 30 per cent. and tutocaine I per $\&$ cent. (lithocaine); and I per cent. novocain with a little adrenalin for local anæsthesia ; $\vec{\circ}$ (5) a scrotal support.

The patient is made to lie on an examination couch or operating table, with a large sand-bag under his buttock. The hydrocele sac is then transilluminated by the lighted torch which is kept in this position until the completion of the operation.

Preparation The skin of the scrotum on the affected side is then sterilized with Patient. surgical spirit, and a suitable spot is selected for the injection of the local anæsthetic, which is performed with the finest hypodermic needle. This preliminary infiltration with local anæsthetic renders all the subsequent procedure painless, and, in addition to being very much appreciated by the patient, avoids any involuntary movements which might lead to such mishaps as the introduction of the aspirating needle into a vein (causing hæmatocele or hæmatoma), or into the testicle itself.

The stiletted lumbar puncture needle is carefully introduced into the middle of the hydrocele sac, and after the stilette is removed all the fluid in the sac should be evacuated into a measured glass flask.

It is very important to remove all the fluid, which is rich in albumin and may "fix" or dilute the sclerosing solution after it has been injected into the sac. Some authorities consider that it is important to wash out the sac with sterile water in order to ensure that no albuminous fluids remain. I am not satisfied that this is necessary, but I am convinced of the advisability of seeing that the sac is completely empty before the injection is undertaken.

Numerous solutions have been injected into the hydrocele sac at various times, e.g., carbolic acid, tincture of iodine, alcohol, and all the sclerosing solutions in vogue to-day. My order of preference, however, would be: (a) Quinine-urethane, Solutions
used. $\quad 2$ to $3 \mathrm{c.cm}$; (b) lithium salicylate 30 per cent. with tutocaine I per cent. (lithocaine), 3 to $4 \mathrm{c.cm}$. The dose of each solution will vary according to the size, consistence, and duration of the sac, the above being the average dose.

After the ro-c.c. Record syringe has been filled with the solution selected for the particular case, it is affixed to the lumbar puncture needle and introduced into the hydrocele sac. The puncture spot is then sealed off with a strip of elastoplast, and the scrotum thoroughly massaged for a few minutes to ensure that the fluid comes in contact with every portion of the sac and its various recesses.

A suspensory scrotal support is applied at the completion of the operation, and the patient dismissed with instructions to report in a week's time for inspection. When seen a week later the scrotum is usually found to be hard and leathery, and in some cases slightly inflamed.

A certain amount of fluid re-collects, and I consider it advantageous to remove this 
by aspiration, using a No. 16 hypodermic needle and a Io-c.c. Record syringe for this purpose. I am not quite convinced that this aspiration is necessary in all cases, but in some, where the scrotum is tense, the evacuation of the fluid would certainly appear to expedite the convalescence.

The patient may complain of a certain amount of pain during and after the injection, but it is never so bad as to keep him away from his usual occupation. Some tenderness on pressure may persist for days or weeks, but this subsides and eventually disappears as the swelling diminishes. In a satisfactory case the cure is completed in from six to eight weeks. The final results are on the whole gratifying, and approximately 75 per cent. of all cases will be cured by this method. I have treated, or have been responsible for the treatment of seventeen cases in private and in hospital practice, with known results. Fourteen of these were cured, and there were three failures, two of which had subsequently to be operated on. The treatment is extremely favourable in children, yielding good end-results, and should be advised for those cases in which the sac does not communicate with the abdominal cavity.

Recently I have treated three further cases by a new method which, although at present in the experimental stage, gives every promise of being superior to the method previously employed. The procedure is the same except that, instead of introducing only a small quantity of the sclerosing solution, the sac is first distended to its fullest capacity with the lithocaine or quinine-urethane solution, whichever has

\section{A New}

Technique. been selected for use. This is allowed to remain for two or three minutes in order to ensure that every part and every crevice of the interior of the sac is subjected to the action of the solution, allowing no portion of the sac to escape its irritating influence.

At the end of this time the sac is evacuated of its contents except for a small amount which is allowed to remain. By this method the entire sac wall is so universally affected that a complete adherence of the parietal and visceral layers of the tunica vaginalis is rendered certain, and, it may be hoped, permanent.

My experience with the use of smaller amounts of solution, even where the recommended massage has been efficiently carried out, has been that certain parts of the interior of the sac may escape the action of the irritant, leading to imperfect effusion of the solution and the formation of one or more loculi.

The causes of failure with either method may be summarized as follows :-

(I) Treatment of unsuitable cases. It has been found that where the sac is of very long standing, grossly distended, fibrotic-the distance between the parietal and visceral layers of the tunica vaginalis being widely separated and stretched, and the walls being firm, thickened and inelastic-a successful issue is doubtful.

(2) Injection of too little solution, or the employment of an unsuitable sclerosing medium.

(3) Incomplete evacuation of the hydrocele fluid, or leaving a loculus untapped. 